\title{
La comunicación y la educación: un proyecto de comunicación educativa
}

The Communication - education relation: an educational Communication project

\author{
Olga Lucía Bedoya ${ }^{1}$ \\ Julián David Vélez Carvajal ${ }^{2}$ \\ Victoria Eugenia Valencia ${ }^{3}$
}

Bedoya, Olga. Vélez C, Julián D. Valencia, Victoria E miradas $\mathrm{N}^{\circ} 14$ - 2016. ISSN: 0122 994X Págs 146 - 163

Recepción: Junio 18 de 2016

Aprobación: Octubre 03 de 2016

Publicación: Diciembre 20 de 2016

\section{Resumen}

En este artículo se da cuenta del proceso de observación de segundo orden realizado sobre la investigación realizada en la maestría en Comunicación Educativa de la Universidad Tecnológica de Pereira, durante el periodo de tiempo 2009 - 2014. Este proceso se realizó con el propósito de distinguir los esquemas de referencia teóricos y metodológicos, desde los cuales se construye conocimiento sobre la relación entre comunicación y educación.

Palabras Clave: Comunicación, educación, cultura, observación de segundo orden, teoría, metodología, objeto de estudio.
Abstract:
This article describes the second order observation exercises concerning to the Edu - Communication master's investigation during 2009-2014. The purpose is to differentiate the reference schemas in theoretical and methodological terms, to construct knowledge about the Communication - education relation.
Key Words: Communication, education, culture, second order observation, theory, methodology
1 Doctora en Ciencias Políticas, Directora de la Maestría en Comunicación Educativa de la Universidad Tecnológica de Pereira. olbedoya@utp.edu.co
2 Doctor en Ciencias de la Educación, Docente Titular y director del grupo de investigación en Cultura, Comunicación y Educación - COEDU- de la Universidad Pontificia Bolivariana de Montería. julian.velezc@upb.edu.co
3 Doctora en Ciencias de la Educación y docente de primaria. victoriavalencia@utp.edu.co 


\section{Introducción}

El presente artículo aborda los esquemas observacionales emergentes a los trabajos de investigación realizados por los integrantes de la Maestría en Comunicación Educativa de la Universidad Tecnológica de Pereira. Dicho ejercicio investigativo se realiza con el propósito de reconocer las distinciones desde las cuáles se construyen los objetos de estudio atinentes a la relación entre comunicación y educación.

Observar distinciones nos ubica en una perspectiva que reconoce al conocimiento como un ingenio discursivo que se construye según sean las circunstancias en las que se encuentre el observador; lo cual nos acerca a una perspectiva constructivista del proceso de conocer que, además de reconocer el carácter generativo de los procesos cognitivos, demanda la capacidad y la disposición de hacer consciencia de los puntos de vista particulares en los que nos ubicamos.

La maestría en Comunicación Educativa, en el marco del ejercicio investigativo, construye una concepción específica no sólo sobre las categorías de comunicación y educación; sino sobre las relaciones de continuidad que se establecen entre éstas. Dicha especificidad se ve reflejada en las preguntas de enlace que utilizamos para nuestra reflexión académica: ¿Cómo educa lo que comunicamos? ¿Es la explicitud de la intencionalidad educativa una condición sine qua non para el reconocimiento del carácter educativo de cualquier proceso social? O ¿Todos los procesos sociales son susceptibles de educar independientemente de que haya, o no, intencionalidad educativa?

Los anteriores interrogantes son transversales a cualquier apuesta por construir conocimiento sobre la relación entre comunicación y educación; pero las respuestas que se dan a estos interrogantes y la manera en que éstas se construyen, se constituyen en un aspecto específico que es relativo a un punto de vista particular. $Y$ tal especificidad se hace posible gracias a la variedad de teorías, metodologías y objetos de estudio que, al ser entrecruzados, fungen como respuestas a los cuestionamientos mencionados en el párrafo precedente.

La maestría en Comunicación Educativa cuenta con una estructura investigativa que permite distinguir delimitaciones acerca de las perspectivas teóricas, los enfoques metodológicos y los objetos de estudio suscritos por este programa de formación. Siguiendo los requerimientos del Sistema Nacional de Ciencia, Tecnología e Innovación de Colombia - COLCIENCIAS-, este programa de formación se estructura a partir de tres líneas de investigación y un grupo de investigación que se encarga de generar proyectos con los subsecuentes productos asociados al ejercicio investigativo ${ }^{4}$.

Si bien un análisis de las líneas de investigación y de los productos de generación de nuevo conocimiento, permite hacer algunas inferencias con respecto a los puntos de vista desde los cuales se construye conocimiento en torno a la relación entre comunicación y educación; se consideró que este procedimiento no aportaba la profundidad requerida para distinguir los esquemas de referencia de la maestría en Comunicación Educativa sobre este aspecto.

Desde el punto de vista de la teoría de sistemas del sociólogo alemán Niklas Luhmann, la observación de segundo orden se constituye en un procedimiento

\footnotetext{
4 El grupo de investigación se denomina Comunicación Educativa - GICE-; y las líneas de investigación, son las siguientes: a) La comunicación y la educación en los procesos de transformación cultural; b) La educación en los medios y los medios en la educación; y c) Nuevas tecnologías para la educación.
} 
que permite observar las distinciones de las que partimos para realizar nuestras propias observaciones (Luhmann, 2005). Dichas distinciones, no son otra cosa que los esquemas de referencia que fungen como marcos interpretativos para la construcción de conocimiento sobre los objetos de estudio abordados.

Ahora bien, en el caso de la construcción de conocimiento desde el punto de vista del método científico ¿Qué es lo que permite distinguir la construcción de dichos esquemas de referencia? Desde la sociología serían diversas las categorías que posibilitarían la distinción de los esquemas de referencia en los procesos de construcción de conocimiento: filiación política, nivel educativo, clase social; etc. No obstante, como nuestra apuesta consiste en conocer la manera en que se elabora conocimiento sobre la comunicación educativa desde los campos teóricos y sus respectivas conexiones con los objetos de estudio, se definió que las categorías para hacer seguimiento al proceso en cuestión serían las siguientes: a) perspectivas teóricas; b) enfoques metodológicos y c) objetos de estudio.

Por lo tanto, se propuso la siguiente pregunta de investigación: ¿Es posible distinguir los esquemas de referencia en cuanto a teorías, metodologías y objetos de estudio, presentes en los procesos de investigación realizados por el grupo de investigación de la maestría en Comunicación Educativa, durante el periodo de tiempo comprendido entre los años 2009 y 2014 ?

Un estudio de estas características, además de permitir la realización de un proceso de auto - observación por parte de los integrantes del grupo de investigación de la maestría en Comunicación Educativa, posibilita actualizar los aportes que desde este programa de formación y su respectivo grupo de investigación, se están realizando al campo de conocimiento atinente a la relación entre comunicación y educación.

\section{La observación de segundo orden y la distinción de esquemas de referencia}

Para la perspectiva teórica de este proyecto de investigación, tomamos como punto de referencia el concepto de observación de segundo orden propuesto por el teórico alemán Niklas Luhmann (2005). Lo planteado bajo esta mirada, nos permite dimensionar, de manera adecuada, la pretensión de realizar un trabajo estas características.

Nuestro propósito no es el de hacer una mera relación de los trabajos de investigación que se han realizado en el marco de la maestría en Comunicación Educativa de la Universidad Tecnológica de Pereira en un determinado periodo de tiempo. Pretendemos apuntar a la posibilidad de dimensionar la manera en que los autores de los diferentes trabajos seleccionados abordan los objetos de estudio que son investigados. Observar observaciones es una afirmación que puede dar cuenta del procedimiento que realizamos para la materialización de este trabajo. Y justamente el teórico Luhmann (2005), deviene esta afirmación en concepto bajo la óptica de su teoría de los sistemas sociales.

Según este autor, la observación consiste en un procesamiento de selecciones que derivan en una distinción entre aquello que se actualiza y aquello que se deja en la opacidad en el mencionado proceso (Luhmann, La realidad de los medios de masas , 1995). Esto quiere decir que la observación, tal como la entiende el autor de referencia, no puede dar cuenta omniabarcante de lo observado; por el contrario, parte de un punto ciego gracias al cual puede ver unas cosas, pero no otras. 
En un primer umbral, la observación no hace conciencia de las distinciones que realiza entre actualidad y potencialidad y, por tanto, no distingue el punto ciego del que parte y que permite delimitar lo construido en términos de dicha observación. Esto es lo que Luhmann denomina observación de primer orden (1995), atribuyéndole como principal característica el que, al estar totalmente focalizado en lo observado, el observador no está en capacidad de distinguir las distinciones de partida. (Luhmann, 1995).

¿El proceso del observar se agota en lo descrito en las líneas precedentes? O ¿Es posible realizar una observación que permita distinguir el punto ciego del que se parte? ¿Es posible distinguir distinciones? ¿Observar observaciones? Luhmann, plantea un segundo umbral de observación en el que es factible observar la manera en que observamos. Esto es lo que el autor en mención denomina observación de segundo orden. (Luhmann, 1995).

En términos de este autor, la observación de segundo orden es como una observación de primer orden que tiene como rasgo fundamental observar las distinciones de las que partimos para realizar nuestras observaciones. (Luhmann, 1995). Por tanto, este proceso de segundo orden permitiría dar cuenta de la manera en que se construye la realidad a través de parcelaciones de la misma.

En un sentido operativo, la observación como proceso discriminante supone seleccionar sólo unos aspectos de lo observado, dejando por fuera otras posibilidades. Lo no seleccionado no es inexistente, por el contrario, cobra suma importancia al momento de realizar una observación de segundo orden, pues da información importante acerca de los puntos ciegos que suscitan los diferentes esquemas de referencia de los observadores.
Es como si el observador contara con múltiples posibilidades, de las cuales solo puede seleccionar algunas según se lo permita el punto ciego del que parte. Distinguir la relación entre las múltiples posibilidades seleccionables y la selección realizada por el observador es altamente significativo por cuanto permite comprender la manera en que la realidad es construida a través de la observación en tanto proceso discriminante.

Los conceptos de medio y forma desarrollados por Luhmann (1995), permiten comprender este proceso. El medio es como una especie de compilación de unidades dispersas que son susceptibles de ser relacionadas y combinadas de diferentes maneras. Dichas unidades dispersas, no oponen resistencia a las múltiples posibilidades de combinación, por lo que potencian la posibilidad de darle forma a "algo" en términos infinitos. Cómo se generan unas combinaciones y no otras, es cuestión de los esquemas de referencia suscitados por el punto ciego del observador.

La forma es, entonces, el resultado de la particular cohesión y estructuración que asumen las unidades dispersas, según sean las disposiciones del observador. Es de aclarar que las posibles maneras de combinar las formas son bastante altas en términos cuantitativos. El alfabeto y las palabras que construimos a partir de la combinación de las consonantes y vocales que lo constituyen es un buen ejemplo para comprender la relación medio / forma. Las 27 letras del abecedario se constituyen en el medio y las palabras en las posibles formas que son el resultado de los millones de combinaciones que son susceptibles de realizarse.

La forma contiene al medio, en la medida en que lo actualiza y le da existencia; por lo tanto, puede constituirse en medio en 
la medida en que funja como objeto de observación por parte de un observador. Esto aplica, de manera especial, para el caso de la observación de segundo orden en la medida en que el observador se focaliza en las formas construidas por otros observadores. Una concepción tal sobre la observación, tiene profundas implicaciones en cuanto a lo que por ella se ha concebido tradicionalmente en la cultura occidental:

- La observación se realiza en el mundo por parte observadores concretos.

- Al ser realizada por observadores concretos, la observación supone el trazo de unos límites específicos que suscitan la relevancia de unas unidades y la opacidad de otras.

- La observación, entonces, no da cuenta del mundo tal como este se nos muestra, sino de los esquemas de referencia suscitados por los puntos ciegos del observador.

- De lo anterior deriva el carácter significativo de la observación de segundo orden, pues permite distinguir las diversas maneras en que se procesan las selecciones del observador.

- El hacer conciencia de que en el proceso del observar quedan aspectos en la opacidad, sugiere reconocer la importancia de éstos, en la medida en que dan información importante acerca de la manera en que el observador construye la realidad.

En este orden de ideas ¿Es posible realizar una observación de segundo orden de los trabajos de investigación realizados por el grupo de investigación de la maestría en Comunicación Educativa de la Universidad Tecnológica de Pereira? ¿Se puede distinguir lo que funge como medio para que los investigadores del grupo en mención hagan sus respectivas selecciones con el propósito de construir su trabajo de investigación?
Con respecto a la primera pregunta, aseveramos que la importancia de este trabajo radica en que permite reconocer los esquemas de referencia desde los cuáles se construyen los diferentes trabajos de investigación que aportan a la construcción de la relación entre cultura, comunicación y educación como campo de conocimiento emergente. En relación con la segunda pregunta, podemos afirmar que el medio tiene una constitución tripartita: 1) las diferentes teorías de la comunicación, la educación y la cultura; 2) la línea y sublíneas de investigación del programa en cuestión; 3) los diferentes objetos de estudio pertinentes al campo de la comunicación educativa. Estos tres referentes cuentan con unidades dispersas que al ser combinadas de múltiples formas, posibilitan la concretización de los diferentes trabajos de grado referidos en páginas precedentes.

\section{Distinción de otras distinciones: algunos antecedentes}

El presente estado del arte permite conocer los principales hallazgos relacionados con la comunicación educativa y la observación de segundo orden. La información recolectada y posteriormente analizada se seleccionó de las bases de datos Communication and Mass Media Complete, E book Mc Graw Hill y EBSCO Host, proporcionadas por la Universidad Pontificia Bolivariana y por la Universidad Tecnológica de Pereira.

En el proceso de búsqueda, se encontraron cuatro artículos que hacen referencia a la observación de segundo orden pero desde el ámbito de las matemáticas, la economía y las finanzas; por lo cual fue necesario acudir a otros repositorios de bibliotecas universitarias, que aportaron trabajos abordados desde otras perspectivas; en medio de esa búsqueda se encontraron dos investigaciones de la Universidad 
Tecnológica de Pereira, que articulan la comunicación con la observación de segundo orden. Por esta razón, se hace necesario presentar los hallazgos en dos grupos para poder analizarlos particularmente.

\section{Grupo I: Matemáticas, Economía y Finanzas}

El primer artículo se denomina "Dificultades y paradojas de la observación de segundo orden: Reflexiones en torno al cálculo de la forma" (Salgado, 2012), quien centra su reflexión sobre los postulados de George Spencer Brown, utilizando la observación de segundo orden desde la teoría de los sistemas sociales que propone Niklas Luhmann, para demostrar cómo pueden ser observadas las operaciones de observación.

Se destacan las posibilidades que se abren para las ciencias sociales con el uso de la forma como estrategia de observación, y a partir de esto se establecen once tesis en las que se enfatiza que la observación es una construcción del observador, es un manejo de distinciones contextualizadas, es indeterminada, paradójica y arbitraria y no persigue la verdad, entre otras. De igual manera, se redefine la teoría de la observación de segundo orden desde el enfoque de Luhmann.

De igual manera, el autor sostiene que la observación de segundo orden no puede ni debe ser crítica, ya que como lo expresa Luhmann (1995), "no puede separar, distinguir, juzgar o condenar" como se hace con otro tipo de teorías sociales.

El segundo artículo intitulado: "Burbujas especulativas y crisis financieras: Una aproximación neofuncionalista", (García, 2015), sustenta su reflexión en la utilización de la diferenciación funcional y la observación de segundo orden en torno a la dinámica de los mercados financieros, específicamente, en lo relacionado con el fenómeno de la burbuja especulativa que origina la crisis financiera, y es abordado desde tres dimensiones: los análisis económicos de la crisis financiera, los «animal spirits», la sociología económica de la burbuja financiera y el enfoque sociológico alternativo.

El autor sostiene que el factor clave de la crisis es el exceso de confianza en las actividades financieras sin pronosticar las consecuencias a largo plazo, es decir, que son fenómenos esencialmente psicosociales y que un elemento explicativo fundamental de la formación de la burbuja especulativa que ha desembocado en la crisis financiera, son las redes sociales y las conexiones personales establecidas por las élites financieras, que configuran un mundo social y culturalmente cerrado, según expone García Blanco.

Cabe anotar que la observación de segundo orden permite hacer el análisis de la lógica en la que operan los mercados financieros, es decir, que esta forma de observación es más radical puesto que la dinámica (de estos mercados), enmarca sus resultados en una especie de particular patología social que desemboca en densas redes sociales y suele conducir al desarrollo de conductas especulativas.

El documento "Observando las finanzas como una red de observaciones: Un comentario a Esposito" (Stark, 2014), presenta una contribución a la teoría de la observación a partir de los aportes que hace Helena Esposito en su artículo: "Circularidades económicas y observación de segundo orden: La realidad de las calificaciones." Basado en las reflexiones que le suscita este artículo y en los postulados de Niklas Luhmann; el autor refuerza la idea de que la sociedad se constituye por el proceso de observación mutua. Por eso es preciso, según él, aplicar 
la teoría de la observación al campo de las finanzas y al estudio de las calificaciones crediticias, lo que demuestra que las observaciones son parte constitutiva de la economía.

Finalmente, en el artículo intitulado: "Comentario de cierre. La observación de segundo orden como perspectiva sociológica y financiera" (Rodríguez, 2014), se hace también un análisis sobre las características y límites de la observación de segundo orden teniendo en cuenta la discusión entre Helena Esposito y David Stark, investigadores mencionados anteriormente.

\section{Grupo II: Comunicación Educativa}

La Universidad Tecnológica de Pereira hizo una recopilación y análisis de los trabajos de grado de los estudiantes de la Maestría en Comunicación Educativa de esta institución a través de la publicación de dos libros: Modos de Ver I, que va del año 1990 hasta el 2002 (Bedoya, 2002), y Modos de Ver II, del 2003 al 2007 (Bedoya, Modos de ver II. Los sentidos de la comunicación Educativa, 2008). Estas investigaciones se convierten en referente fundamental puesto que en ellas se configuran los elementos de análisis que sirven como apoyo al trabajo de documentación de esta propuesta.

Se destaca, entonces, el estado del arte que pone en escena las tendencias de investigación emergentes en la Universidad Tecnológica de Pereira, acompañado de un ejercicio sistemático de comparación en torno a los esquemas observacionales, marcos teóricos y metodológicos que delimitan la comunicación educativa no solo como concepto sino como campo de estudio.

Además, se hace un aporte a la relación que existe entre comunicación y educación que establece la maestría en Comunicación
Educativa, centrada en sus líneas de investigación. Las reflexiones surgen del análisis de la comunicación educativa desde diversas perspectivas teóricas y metodológicas tomando como referente a Niklas Luhmann y como base la teoría de los sistemas sociales.

De acuerdo con los trabajos anteriormente mencionados, se puede evidenciar que existe relación entre las investigaciones en el campo de la educación, las matemáticas, las finanzas y la economía, en la medida en que realizan sus reflexiones desde la teoría de Luhmann, especificando que la observación es un fenómeno eminentemente constructivo donde existen procesos comunicativos de observaciones que se ocupan de observar observadores.

No obstante, se puede evidenciar que en el ámbito de la relación entre comunicación y educación, solo se encuentran los trabajos hechos desde la Universidad Tecnológica de Pereira. Lo cual, además de aportar a la consolidación de las líneas y sublíneas de investigación del programa en mención; otorga posibilidades comparativas con los esquemas de referencia que se vienen desarrollando en los diferentes escenarios académicos.

De igual manera, cada uno de los documentos encontrados aportan significativamente a esta investigación y permiten constatar que, desde la observación de segundo orden, se puede aportar al campo de conocimiento que nos interesa: La comunicación educativa.

\section{Materiales y métodos}

El presente proyecto se inscribe dentro de lo que se denomina investigación documental; por cuanto se pretenden distinguir los esquemas de referencia en cuanto a teorías, metodologías y objetos de estudio, presentes en los trabajos de 
investigación realizados en el marco de la Maestría en Comunicación Educativa de la Universidad Tecnológica de Pereira. Según el Manual de Trabajos de Grado de Especialización, Maestría y Tesis Doctorales (UPEL), "la investigación documental es el estudio de problemas con el propósito de ampliar y profundizar el conocimiento de su naturaleza con apoyo, principalmente, de trabajos previos, información y datos divulgados por medios impresos, audiovisuales o electrónicos" (2006). En este caso, los trabajos previos, son los diferentes proyectos de investigación realizados por el grupo de investigación en mención durante el periodo de tiempo 2009 - 2014 .

Fuentes de recolección de la información:

Las fuentes de recolección de la información son de carácter primario y se encuentran constituidas por los trabajos de investigación adscritos a la maestría en Comunicación Educativa de la Universidad Tecnológica de Pereira, durante el periodo de tiempo: 2009 - 2014 .

\section{Instrumentos de recolección de la información:}

Como la información es de tipo documental, se accedió a cada uno de los trabajos de investigación en versión digital; después de organizar un archivo con dichos trabajos, se procedió al diseño de una matriz de observación en la que se realizó un ejercicio analítico de cada uno de las investigaciones. La matriz, se estructuró a partir de las siguientes categorías de análisis: 1) pregunta o problema de investigación; 2) marco teórico; 3) metodología; 4) objetivos; 5) conclusiones.

\section{Proceso de análisis y síntesis de la información:}

Este procedimiento, se desarrolló en tres fases:

Fase 1. Procedimiento analítico: en este momento se tomó cada uno de los trabajos y se sometió a un proceso de análisis exhaustivo a partir de las categorías: teorías, metodologías y objetos de estudio. Este procedimiento permitió la generación de gráficas que posibilitaron visualizar una cartografía general con respecto a los trabajos de investigación de la maestría en Comunicación Educativa.

Fase 2. Comparación: como la matriz de observación se diseñó en formato Excel, se hizo uso de la opción de filtrado con el propósito de realizar un proceso comparativo tomando como criterio las categorías: teoría, metodología y objetos de estudio. Este procedimiento permitió identificar tendencias con respecto a las categorías en mención. Igualmente, se pudieron identificar residuos, los cuales se constituyeron en una fuente prolija para la distinción de los esquemas de referencia presentes en los trabajos de investigación en mención.

Fase 3. Construcción de esquemas categoriales: el proceso analítico y comparativo elaborado en las dos fases anteriores, sentó las bases para la elaboración de esquemas categoriales que fueron de dos tipos:

a. Esquemas de observación emergentes: estos esquemas dan cuenta de información importante, de tipo sincrónico, acerca de las características de la investigación en la maestría en Comunicación Educativa de la Universidad Tecnológica de Pereira.

b. Esquemas de observación referenciales: estos esquemas son de tipo diacrónico y 
dan cuenta de las intensidades temporales y tendencias temáticas de las investigaciones durante todo el periodo de tiempo seleccionado.

El cruce entre los esquemas de observación emergentes y los esquemas de observación referenciales apuntaron a la construcción de un esquema de tipo general que permitió allegarse a los esquemas de referencia en cuanto a teorías, metodologías y objetos de estudio presentes en los trabajos de investigación realizados al interior de la maestría en Comunicación Educativa de la Universidad Tecnológica de Pereira.

4. Interpretación: La interpretación se hizo a partir del cruce entre pregunta de investigación, teoría, metodología y dato. En este proceso se realizó la discusión de los hallazgos haciendo uso de la teoría de la observación de segundo orden desarrollada por el sociólogo alemán Niklas Luhmann.

\section{Análisis y discusión de resultados}

Se presenta en este apartado la condensación de los resultados de esta investigación, con su respectivo análisis. Para ello, en primera instancia, se actualizarán los esquemas de observación referenciales y emergentes al proceso de investigación en mención. Luego, se dará cuenta de la constitución tripartita del medio en el marco de la construcción de conocimiento por parte del grupo de investigación de la maestría en Comunicación Educativa.

\section{Esquemas de observación referenciales}

El proceso de análisis y síntesis de la información recolectada permitió la distinción de esquemas observacionales de tipo referencial. Estos esquemas se caracterizan por permitir evidenciar las intensidades temporales y temáticas de la investigación realizada por los integrantes de la maestría en Comunicación Educativa.

\section{Intensidad temporal de las investigaciones}

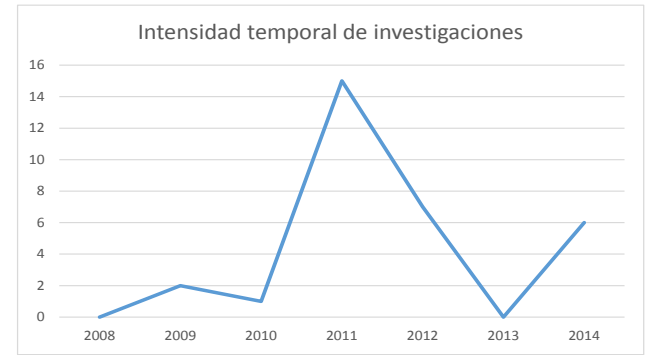

Fuente: propia

En el marco del periodo de tiempo que se seleccionó para este proceso de indagación, se evidencia un incremento de la actividad investigativa del grupo de Comunicación Educativa entre los años 2010 y 2014. No obstante, se puede evidenciar que, a excepción del periodo de tiempo correspondiente a 2013, el grupo analizado estuvo activo en cuanto a la creación y ejecución de proyectos de investigación.

La variabilidad que se evidencia en la actividad investigativa del grupo es consecuencia de aspectos tanto internos como externos al grupo de investigación. Desde el punto de vista endógeno, la dinámica depende del número de investigadores que lo integran y de su cronograma en cuanto a los procesos de investigativos; mientras que desde lo exógeno, las prácticas investigativas del grupo dependen de las agendas para la financiación de proyectos de investigación por parte de las vicerrectorías de investigación de las universidades y del Departamento Nacional de Ciencia, Tecnología e Innovación de Colombia COLCIENCIAS-

Este esquema de observación cobra importancia en la medida en que se cruce con los esquemas correspondientes a la intensidad por temas y líneas de 
investigación; por cuanto permite vislumbrar la agenda temática del grupo de investigación como un proceso emergente a los factores endógenos y exógenos de la intensidad temporal.

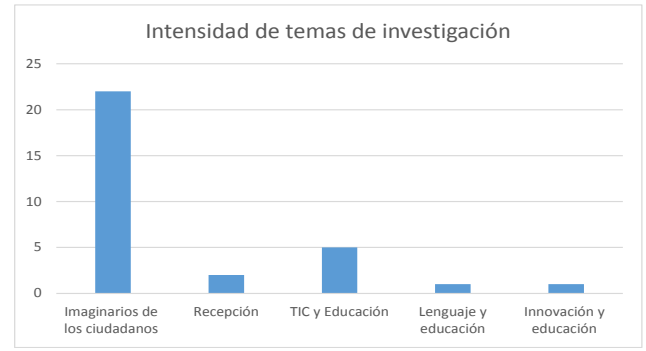

Fuente: propia

La intensidad por temas de investigación evidencia una marcada tendencia entre las categorías asociadas a las relaciones ciudad - comunicación y TIC - educación. Sin embargo, esta supremacía de ambas relaciones se relativiza si se tiene en cuenta que las categorías recepción, lenguaje educación e innovación - educación son conexas a las dos más prominentes, desde el punto de vista teórico y metodológico.

$\mathrm{Si}$ bien el ejercicio distributivo nos permite evidenciar ciertas tendencias, la perspectiva relacional aplicada en el grupo de investigación abordado, permite entrever la correlación existente entre las líneas de investigación propias de la maestría.

\section{Intensidad temática por líneas de investigación}

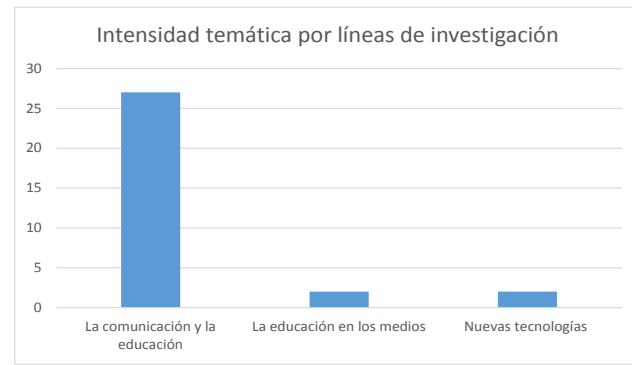

Fuente: propia
Las líneas de investigación guardan una estrecha relación con la intensidad temática; esta última se estructura, precisamente, a partir de las delimitaciones que traen consigo tales líneas. Así, si se comparan las intensidades por temas y líneas de investigación, se puede inferir la correlación entre la tendencia "imaginarios urbanos" y la tendencia "la comunicación y la educación"; lo cual indica las conexidades que también se establecen entre las líneas de investigación del programa.

En este orden de ideas, la correlación entre las intensidades temporal, temática y de líneas de investigación, permite realizar una descripción general del panorama de investigación de la maestría en Comunicación Educativa. Ahora bien ¿Permiten tales intensidades distinguir los esquemas de referencia presentes en la investigación del programa de formación en mención? Los esquemas de observación referidos anteriormente, sirven como pretexto para su distinción; pero no posibilitan alcanzar este propósito de manera cabal; para ello es necesario la construcción de los esquemas de observación emergentes.

\section{Esquemas de observación emergentes}

Esta fase de la investigación es de orden interpretativo, por lo que da cuenta de las relaciones cognitivas que se establecen en el grupo de investigación de la maestría en Comunicación Educativa, al momento de abordar la relación entre cultura, comunicación y educación. Cuando se cruzan los esquemas de observación referenciales y la perspectiva teórica que suscribimos en este proyecto de investigación, se puede establecer que dichos esquemas son de carácter tripartito.

Se había planteado, en apartados precedentes, que los conceptos de medio y forma (Luhmann, El arte de la sociedad, 
2005), ayudan a comprender el proceso de observación de segundo orden. Habíamos dicho, también, que el medio es una compilación de unidades dispersas que se pueden relacionar y combinar de diversas maneras; dichas unidades no oponen resistencia a las posibles alternativas de combinación, lo cual permite la posibilidad de construir "formas" al infinito.

Para el caso de esta investigación, se puede afirmar que el medio lo constituyen las diferentes teorías asociadas a la relación comunicación - educación y cultura; así como las líneas de investigación del posgrado en Comunicación Educativa y los objetos de estudio relacionados; de ahí el carácter tripartito del medio. Ahora bien, de la manera en que se combinen estos tres elementos, depende la consolidación de las "formas", que no son otra cosa que los esquemas de referencia emergentes a la investigación del programa en mención.

A continuación se hará referencia a cada uno de los elementos que constituyen "el medio" para la construcción de formas en el marco de la investigación en la maestría en Comunicación Educativa de la Universidad Tecnológica de Pereira:

\section{Esquema emergente de observación $\mathbf{N}^{\circ}$ 1: Perspectivas teóricas elegidas}

Las categorías abordadas en cada una de las investigaciones realizadas en la maestría en Comunicación Educativa durante el periodo de tiempo seleccionado, dan información importante con respecto a las perspectivas teóricas elegidas en este programa de formación:

\section{Categorías}

\begin{tabular}{|c|c|c|c|c|}
\hline \multicolumn{5}{|c|}{ Categorías conceptuales } \\
\hline 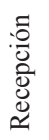 & & Comunicación & $\begin{array}{l}\text { Acción } \\
\text { comunicativa }\end{array}$ & Representación \\
\hline 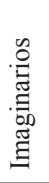 & 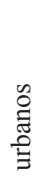 & Signo & Enseñanza & Aprendizaje \\
\hline 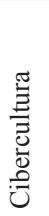 & & Ciberespacio & $\begin{array}{l}\text { Culturas } \\
\text { juveniles }\end{array}$ & Ciudadanía \\
\hline
\end{tabular}

Fuente: propia

Las anteriores categorías son conceptualizadas desde las perspectivas teóricas desarrolladas por diferentes autores provenientes de escuelas de pensamiento de América Latina, América del Norte y Europa. La selección de estas escuelas, da índices acerca de la manera en que se construyen los esquemas de referencia con respecto a las perspectivas teóricas del proceso de investigación abordado en este documento: 


\section{Escuelas de pensamiento}

\begin{tabular}{|c|c|c|c|}
\hline Autor & Categoría abordada & Escuela de Pensamiento & Procedencia \\
\hline $\begin{array}{l}\text { Charles Sanders } \\
\text { Peirce }(1839- \\
1914)\end{array}$ & Representación, Signo & $\begin{array}{l}\text { Phaneroscopia, pensamiento } \\
\text { tríadico, pragmatismo. }\end{array}$ & Estados Unidos \\
\hline $\begin{array}{l}\text { Gilbert Durand } \\
(1921-2012)\end{array}$ & Imaginarios & $\begin{array}{l}\text { Círculo de Eranos. Perspectiva } \\
\text { arquetípica de los imaginarios, } \\
\text { psicoanálisis de Carl Gustav Jung, } \\
\text { epistemología de Gaston Bachelard. }\end{array}$ & Francia \\
\hline $\begin{array}{l}\text { Lev Vigostky } \\
(1896-1934)\end{array}$ & Enseñanza - aprendizaje & $\begin{array}{l}\text { Constructivismo socio - cultural, } \\
\text { psicología histórico - cultural. }\end{array}$ & Antigua Unión Soviética \\
\hline $\begin{array}{l}\text { Umberto Eco } \\
\text { (1932) }\end{array}$ & Signo & Semiótica & Italia \\
\hline $\begin{array}{l}\text { David Ausubel } \\
(1918-2008)\end{array}$ & Aprendizaje significativo & Psicopedagogía cognitiva & Estados Unidos \\
\hline $\begin{array}{l}\text { Jürgen Habermas } \\
\text { (1929) }\end{array}$ & $\begin{array}{l}\text { Acción comunicativa, } \\
\text { ciudadanía }\end{array}$ & Escuela de Frankfurt, teoría crítica & Alemania \\
\hline $\begin{array}{l}\text { Niklas Luhmann } \\
(1927-1998)\end{array}$ & Comunicación & $\begin{array}{l}\text { Funcional - estructuralismo, teoría } \\
\text { de sistemas, cibernética de segundo } \\
\text { orden }\end{array}$ & Alemania \\
\hline $\begin{array}{l}\text { Gregory Bateson } \\
(1904-1980)\end{array}$ & Comunicación & $\begin{array}{l}\text { Escuela de Palo Alto, California, } \\
\text { Estados Unidos; cibernética }\end{array}$ & $\begin{array}{l}\text { Reino Unido - Estados } \\
\text { Unidos }\end{array}$ \\
\hline $\begin{array}{l}\text { Armando Silva } \\
(1948)\end{array}$ & Imaginarios Urbanos & $\begin{array}{l}\text { Imaginarios urbanos. Se basó en } \\
\text { autores como Jacques Derrida, } \\
\text { Henrry Lefevre, Sigmund Freud, } \\
\text { Charles Sanders Peirce, entre otros. }\end{array}$ & Colombia \\
\hline $\begin{array}{l}\text { Néstor García } \\
\text { Canclini } \\
(1938)\end{array}$ & $\begin{array}{l}\text { Consumo cultural, proceso } \\
\text { de hibridación cultural }\end{array}$ & Consumo cultural & Argentina-México. \\
\hline $\begin{array}{l}\text { Guillermo Orozco } \\
\text { Gómez (1954) }\end{array}$ & Recepción & $\begin{array}{l}\text { Recepción activa y múltiples } \\
\text { mediaciones }\end{array}$ & México \\
\hline $\begin{array}{l}\text { Pierre Levy } \\
(1956)\end{array}$ & $\begin{array}{l}\text { Cibercultura - } \\
\text { ciberespacio }\end{array}$ & $\begin{array}{l}\text { Antropología de la relación hombre } \\
\text { - máquina }\end{array}$ & Túnez - Canadá \\
\hline $\begin{array}{l}\text { Alejandro } \\
\text { Piscitelli }\end{array}$ & $\begin{array}{l}\text { Cibercultura - } \\
\text { ciberespacio }\end{array}$ & $\begin{array}{l}\text { Antropología de la relación hombre } \\
\text { - máquina }\end{array}$ & Argentina \\
\hline Carlos Scolari & Narrativas transmedia & $\begin{array}{l}\text { Antropología de la relación hombre } \\
\text { máquina. Planteamientos de } \\
\text { Umberto Eco y Algirdas Greimas. }\end{array}$ & Argentina - España. \\
\hline
\end{tabular}

Fuente: propia

Las diferentes perspectivas teóricas seleccionadas para la realización de los trabajos de investigación de la maestría en Comunicación Educativa, dan información sobre la consolidación de este esquema observacional. A este respecto, se pueden plantear tres tendencias que dan cuenta de éste: a) enfoque constructivista, b) La concepción de los fenómenos sociales como procesos de construcción de realidad; y c) la problematización de los fenómenos educativos y comunicativos desde una perspectiva semiótica y antropológica.
El enfoque constructivista mencionado, no hace referencia a una teoría en particular, sino al paradigma constructivista en calidad de corriente de pensamiento que ha tenido un influjo importante en las diversas disciplinas que constituyen las Ciencias Sociales y Humanas. Si bien existe una variedad considerable de enfoques del constructivismo como paradigma epistemológico: constructivismo radical (Watslawick, 1971), constructivismo genético (Piaget, 1970), constructivismo socio - cultural (Vygotsky, 1981), constructivismo sistémico ( (Luhmann, El arte de la sociedad, 2005); podemos 
afirmar que su premisa radica en reconocer que la realidad es un ingenio discursivo construido por los observadores. Esto da índices importantes para comprender de qué manera se abordan los diferentes fenómenos educativos y comunicativos al interior de la maestría en Comunicación Educativa.

En concomitancia con lo anteriormente dicho, los fenómenos educativos $\mathrm{y}$ comunicativos no se asumen desde perspectivas normativas que indiquen de qué manera tienen que ser los fenómenos; estos se abordan como procesos emergentes que pueden tener múltiples manifestaciones. En la perspectiva trazada por Gregory Bateson (1972), no nos quedamos en la lógica de describir los elementos que integran un fenómeno, sino que procuramos construir y distinguir relaciones entre los diferentes elementos que lo constituyen de tal manera que se pueda alumbrar nuevo conocimiento.

Como se ha planteado en apartados precedentes, si bien la comunicación educativa como campo de conocimiento emergente es objeto de diversos abordajes, en el caso de la maestría abordada, se ha optado por un enfoque semiótico y antropológico para comprender la complejidad de los fenómenos educativos y comunicativos. Este enfoque es coherente con la perspectiva constructivista y la distinción de los mundos posibles en los diferentes fenómenos.

\section{Esquema emergente de observación $\mathrm{N}^{\circ} 2$ : Perspectivas metodológicas implicadas}

La perspectiva metodológica que predomina para la realización de los trabajos de investigación en la maestría en Comunicación Educativa, se fundamenta en el enfoque cualitativo y hermenéutico. Es importante aclarar que tal decisión metodológica, no es consecuencia de algún tipo de perfil definido a priori; es el resultado de la comprensión que se tiene de las implicaciones que traen consigo el constructivismo, los mundos posibles y la perspectiva semiótico - antropológica como esquema de referencia teórico.

Se destaca, en este caso, la apuesta que tiene el grupo de investigación por alcanzar un nivel de coherencia importante en términos epistemológicos, teóricos y metodológicos. Por lo tanto, si la concepción de realidad se fundamenta en el constructivismo, las teorías que se seleccionan como ingenio discursivo para la interpretación de los fenómenos, son coherentes con esta perspectiva y, además, tienen implicaciones metodológicas: selección del enfoque cualitativo - hermenéutico.

Lo anterior incide, también, en la manera en que se construye el dato en un proceso de investigación; una concepción teórico - metodológica como la descrita, implica tomar distancia del carácter distributivo de los enfoques binarios que han primado al interior de la filosofía occidental. Si bien se reconoce la importancia de los procesos analíticos en la construcción de conocimiento, se apuesta por el establecimiento de continuidades entre estos y los procesos sintéticos que permiten la distinción de relaciones entre los diferentes elementos que constituyen un fenómeno.

\section{Esquema de observación emergente $\mathrm{N}^{\circ}$ 3: Objetos de estudio seleccionados}

Los esquemas de observación anteriormente referidos, tienen implicaciones en cuanto a los objetos de estudio seleccionados para llevar a cabo el proceso de investigación en el marco de la maestría en Comunicación Educativa de la Universidad Tecnológica de Pereira. A continuación, se hará referencia a ellos:

- La relación enseñanza aprendizaje como un proceso 
comunicativo

- Imaginarios urbanos

- Recepción de contenidos televisivos

- Lenguaje

- Culturas juveniles

- Imaginarios de salud

- $\quad$ La ciudad

- Video - juegos y educación

- Antropología de las pantallas

- Cibercultura y ciberespacio

Los anteriores objetos de estudio, se caracterizan por sus implicaciones inter y transdisciplinares. Esto quiere decir que se constituyen en objetos que problematizan, de manera transversal, a las ciencias sociales y humanas; de allí que la comunicación educativa como campo de conocimiento emergente, beba en la fuente de diferentes disciplinas como: la comunicación, la educación, la semiología, la antropología, la sociología; entre otras.

\section{Conclusiones}

La observación de los esquemas de referencia asociadosalprocesoinvestigativo de la maestría en Comunicación Educativa de la Universidad Tecnológica de Pereira, permitió establecer que se construye una relación de recursividad entre las perspectivas teóricas, los enfoques metodológicos y la selección de los objetos de estudio. Tal recursividad se da en términos de implicación y de coherencia lógica.

Desde el punto de vista de las implicaciones, la recursividad se evidencia en las múltiples entradas para la delimitación de un problema de investigación. No importa si se inicia por la revisión de teorías o por la intención de profundizar sobre un objeto de estudio específico; lo importante es reconocer que la realización de una selección desde un ángulo particular, tiene implicaciones sobre los demás aspectos del proceso de investigación: abordar un objeto de estudio de una determinada manera tiene implicaciones sobre la perspectiva metodológica y, a su vez, sobre las teorías que fungirán como marco interpretativo del mismo.

De lo anterior deriva la coherencia; si los procesos de selección de teorías, enfoques metodológicos y objetos de estudio son recursivos por cuanto se implican entre ellos mismos, entonces, se hace necesario el establecimiento de coherencia en cuanto al diseño e implementación del proceso de investigación. Por lo tanto, suscribir un enfoque epistemológico específico, lleva a tomar unas decisiones teóricas concretas que traen consigo implicaciones metodológicas.

Los esquemas observacionales emergentes al proceso de investigación realizado, permiten comprender la comunicación educativa como un campo de conocimiento que incorpora la continuidad como un pretexto para la reflexión. Con respecto a los pares de oposición que constituyen las categorías asociadas a los objetos de estudio, el conocimiento no se construye sobre la base de la separación de los elementos que los componen; sino a partir de las posibles conexidades que posibilitan la unidad de los mismos.

$\mathrm{Y}$ es en los límites donde se establecen estas conexidades, donde probablemente se constituyan la ambigüedad y la ambivalencia propias de la unidad de los pares de oposición en cuestión. Se puede afirmar por ejemplo que, en la oposición día / noche, la categoría diurna se hace más relevante cuando se encuentra en la 
porosidad de su transición hacia lo nocturno; luego, se da un proceso de concatenación en el que no se puede distinguir el día de la noche; y es allí donde se pueden construir relaciones emergentes en calidad de nuevo conocimiento. De esta manera se abordan los pares de oposición: escuela / cultura, razón/ emoción, comunicación/ educación, etc., propios de la comunicación educativa como campo de conocimiento emergente.

De lo anterior, deriva la continuidad entre el constructivismo, el enfoque semiótico - antropológico y los mundos posibles como apuestas para la construcción de conocimiento sobre los objetos de estudio propios de la comunicación educativa. Estas tres perspectivas suponen la transición del binarismo a la continuidad en tanto sistemas filosóficos que sustentan la aprehensión de los objetos de conocimiento en el marco de la cultura occidental.

Ahora bien, suscribir la continuidad como categoría principal para la construcción de conocimiento, solo se hace posible si se suscribe la observación de segundo orden como perspectiva teórico - metodológica en los procesos investigativos. Si algo caracteriza a la investigación que se basa en el binarismo, es su negación a distinguir los esquemas de referencia desde los que parte para la construcción de conocimiento, por cuanto se asume que existen verdades pre - establecidas. Entre tanto, al reconocer el ingenio humano como el pilar para la construcción de mundos posibles, la continuidad requiere de la observación de los esquemas de referencia, de tal manera que se puedan distinguir las lógicas a partir de las cuales conocemos e interpretamos el mundo.

En este orden de ideas, la maestría abordada aporta a la comunicación educativa de la siguiente manera: desde el punto de vista epistemológico, a partir de la incorporación del constructivismo, la continuidad y la cibernética de segundo orden; desde el punto de vista teórico, a partir de la selección de teorías con apuestas semióticas y antropológicas; y con respecto a lo metodológico, desde la implementación de enfoques relacionales para el abordaje de los objetos de estudio.

\section{Referencia bibliográfica}

Arango, Bustamante, D. (2011). Imaginarios de una Medellín en blanco y negro ciudadanos de hoy leyendo a los ciudadanos de ayer: fotografías del centro de Medellín. (Tesis inédita de Maestría). Universidad Tecnológica de Pereira.

Bedoya, O. (2002). Modos de ver I. Los sentidos de la Comunicación Educativa. Pereira: Editorial Papiro.

Bedoya, O. (2008). Modos de ver II. Los sentidos de la comunicación Educativa. Pereira: Publiprint.

Botero Escobar, N. (2011). Cibernautas y la Medellín imaginada: entre el ethos y el tanathos. (Tesis inédita de Maestría). Universidad Tecnológica de Pereira.

Botero Girón, C. (2011). Discusión de la metáfora como terceridad en los procesos de comunicación de la ciencia, la tecnología y la innovación en la Universidad Tecnológica de Pereira. (Tesis inédita de Maestría). Universidad Tecnológica de Pereira.

Calle Álvarez, D. (2011). Medellín crónicas: una mirada desde sus imaginarios urbanos. (Tesis inédita de Maestría). Universidad Tecnológica de Pereira.

Cardona Cano, C y Álvarez Moreno, M. (2014). Medellín imaginada desde la televisión. Los imaginarios en la serie de televisión: Muchachos a lo bien. (Tesis inédita de Maestría). Universidad Tecnológica de Pereira. 
Castaño Galvis, C. (2014). Pereira, transición de gran ciudad a desarrollo y progreso. (Tesis inédita de Maestría). Universidad Tecnológica de Pereira.

Castaño Rico, S. (2012). Imaginarios religiosos de Medellín con respecto a las imágenes presentes en las familias. (Tesis inédita de maestría). Universidad Tecnológica de Pereira.

Concha Ocampo, E. (2014). Macroproyecto Pereira imaginada: croquis ciudadanos. Caso imaginarios urbanos con aroma de café: puente de Pereira entre el Éxito y el Hotel Camino Real. (Tesis inédita de Maestría). Universidad Tecnológica de Pereira.

Córdoba González, S (2011). Medellín en subjetiva: visión de los ciber/youtube/anos. Estudio de caso: contribución a los archivos urbanos. (Tesis inédita de Maestría). Universidad Tecnológica de Pereira.

Cruz Arizmendi, G. (2012). El mundo perdido de la realidad. (Tesis inédita de Maestría). Universidad Tecnológica de Pereira.

Fernando, S. (2012). Dificultades y paradojas de la observación de segundo orden: reflexiones en torno al cálculo de la forma . Revista MAD - Universidad de Chile, 15-33.

García, J. (2015). Burbujas especulativas y crisis financieras. Una aproximación neofuncionalista. Revista Española de Investigaciones Sociológicas , 71-88.

García, L. (2011). De lo azul a lo cálido y popular. (Tesis inédita de Maestría). Universidad Tecnológica de Pereira.

García Loaiza, E. (2009). Análisis de la competencia televisiva en la interacción maestro - estudiante de básica secundaria y su impacto en la audiencia adolescente televisiva. (Tesis inédita de Maestría). Universidad Tecnológica de Pereira.
Gil Correa, D. (2015). Consideraciones sobre los procesos de recepción en estudiantes de grado décimo del Instituto Agropecuario Veracruz de Santa Rosa de Cabal, Risaralda. (Tesis inédita de Maestría). Universidad Tecnológica de Pereira

Gómez, Rubiano, C. (2011). Pereira imaginada fase II 2009-2014 ¿La ciudad del deseo o la ciudad del corazón partió? (Tesis inédita de Maestría). Universidad Tecnológica de Pereira.

Herrera Díaz, N. (2012). Imaginarios urbanos en la literatura: las novelas como fuente de reconstrucción de los imaginarios sobre Medellín. Caso: La ciudad de todos los adioses de César Alzate Vargas, El dedo índice de Mao de Juan Diego Mejía y Basura de Héctor Abad Faciolince. (Tesis inédita de Maestría). Universidad Tecnológica de Pereira.

Hoyos Morales, J. (2010). Identificación de categorías toulminianas en clase de filosofía a nivel de educación media con nuevas tecnologías. (Tesis inédita de Maestría). Universidad Tecnológica de Pereira.

Hurtado, Tabares, L. (2011). Construcción de ciudad desde la temporalidad plural en transporte público. Pereira imaginada 2009 - 2014. (Tesis inédita de Maestría). Universidad Tecnológica de Pereira.

Lopera Agudelo, A. (2011). Imaginarios urbanos (de ciudad), el cine de ficción realizado en Medellín de 2000 a 2010. (Tesis inédita de Maestría). Universidad Tecnológica de Pereira.

López Montoya, M y Vega González, F. (2012). Emergencia de la función de innovación en la universidad: el proceso de la Universidad Tecnológica de Pereira. (Tesis inédita de Maestría). Universidad Tecnológica de Pereira. 
Llamosa Pérez, V. (2011). Modelos de consumo. El caso de las mujeres protagonistas de la telenovela las muñecas de la mafia. (Tesis inédita de Maestría). Universidad Tecnológica de Pereira.

Muñoz Guzmán, C. (2014). El paisaje cultural cafetero: una encrucijada entre la sostenibilidad y un futuro amenazado. (Tesis inédita de Maestría). Universidad Tecnológica de Pereira.

Muñoz Rincón, D. (2012). Medellín, un recorrido sobre lo oculto en crónicas y reportajes. (Tesis inédita de Maestría). Universidad Tecnológica de Pereira.

Luhmann, N. (1995). La realidad de los medios de masas. Barcelona: Anthropos.

Luhmann, N. (2005). El arte de la sociedad . México: Editorial Herder .

Olga, B. (2008). Modos de ver II. Los sentidos de la Comunicación Educativa. Pereira: Publiprint.

Ospina Lozada, M. (2011). Pereira imaginada 2009 - 2014. Rutinas ciudadanas: una mirada comunicativa de los imaginarios urbanos en el espacio virtual de los jóvenes entre 12 y 16 años de la Institución Educativa Ciudadela Cuba. (Tesis inédita de Maestría). Universidad Tecnológica de Pereira.

Perdomo Rodas, A. (2014). Emprendedor $=$ comunicador de ideas: imaginarios visibilizados en los emprendedores de Parquesoft Pereira. (Tesis inédita de Maestría). Universidad Tecnológica de Pereira.

Piaget, J. (1970). Psicología y epistemología. Barcelona: Ariel.
Piedrahita Bedoya, F. (2011). La radio y los imaginarios urbanos en Medellín: de lo semiótico y lo ideológico a la configuración del imaginario social del paisa. Caso de las noticias de Radio Paisa. (Tesis inédita de Maestría). Universidad Tecnológica de Pereira.

Ramos Olaya, M. (2012). Cuerpo imaginado: objeto de ensoñaciones y deseos. (Tesis inédita de Maestría). Universidad Tecnológica de Pereira.

Rodríguez, D. (2014). Comentario de cierre. La observación de segundo orden como perspectiva sociológica y financiera. Revista - MAD - Universidad de Chile, 6166.

Rojas Hurtado, A. Jiménez, L. Peña Osorio, D. (2014). Macroproyecto: validación de propuesta educativa con investigación basada en diseño. (Tesis inédita de Maestría). Universidad Tecnológica de Pereira.

Salgado, F. (2012). Dificultades y paradojas de la observación de segundo orden: reflexiones en torno al cálculo de la forma. Revista - MAD- Universidad de Chile, 15-33.

Sánchez Muñoz, C. (2009). La palabra de la radio al aula: formación de maestros que atienden estudiantes con discapacidad visual en las aulas integradoras. El caso de la Institución Educativa Pablo Emilio Cardona. (Tesis inédita de Maestría). Universidad Tecnológica de Pereira.

Stark, D. (2014). Observando las finanzas como una red de observaciones: un comentario a Esposito. Revista - MADUniversidad de Chile, 25-38.

Vásquez, Murillo, R. (2011). Marcas Ciudadanas: Una mirada al gusto de los pereiranos. (Tesis inédita de Maestría). Universidad Tecnológica de Pereira. 
Velásquez Bonilla, N. (2012). Imaginarios de ciudad saludable. (Tesis inédita de Maestría). Universidad Tecnológica de Pereira.

Vygotsky, L. (1981). Pensamiento y lenguaje. España: Paidos.

Watslawick, P. (1971). ¿Es real la realidad? Confusión, desinformación, comunicación. Barcelona: Editorial Herder . 